\title{
A Theory of Radar Reflections From a Rough Moon ${ }^{1}$
}

\author{
Donald F. Winter
}

\section{Contribution from Gordon McKay Laboratory, Harvard University, and Applied Research Laboratory, Sylvania Electronic Systems}

(Received July 10, 1961; revised November 13, 1961)

\begin{abstract}
It is proposed in this paper that the total lunar echo at any instant of time is comprised of two components, which are the result of scattering from two general types of terrain features. On the basis of this model, the average theoretical backscattered power flux at the receiver is derived as a function of time for the case of short pulse transmission. The radar cross section of the moon is also calculated. Several comparisons are made between the theory and experimental results obtained with a variety of equipment parameters.
\end{abstract}

\section{Introduction}

More than a quarter century ago experimenters initiated efforts to establish radar contact with the moon. It was not until 1946 that radar equipment had been improved to the point where positive results were obtainable. Since that time the problem of radar reflections from the moon has been subject to extensive investigation by several experimental groups and theoretical workers both here and abroad.

Among the first theoreticians to report their findings in this general area were Grieg, Metzger, and Waer [1948]. Although only continuous wave transmission was considered, the total transmission loss over the earth-moon-earth path was derived for both the smooth and the perfectly diffuse lunar surface models.

During the past few years several experimental groups have looked for moon echoes using pulse transmission at a variety of frequencies. Among these investigations are those reported by Trexler [1958], Yaplee et al. [1958], Hughes [1960], Leadabrand et al. [1960], and Pettengill [1960, 1961]. Adaptations of neither the smooth nor the diffuse moon models could account for the shape of the returns using short transmitted pulses. This disparity is clearly illustrated in figure 1 which presents experimental results obtained by Pettengill [1961] at a frequency of $440 \mathrm{Mc} / \mathrm{s}$ using a transmitted pulse duration $(\tau)$ of $100 \mu \mathrm{sec}$. If the moon scattered radiation like a smooth sphere then the echo would consist almost entirely of a pulse return with a duration of the order of $100 \mu \mathrm{sec}$. In the other extreme, if the lunar surface were a completely diffuse reflector at radar wavelengths then the initial rapid rise of the return would be followed by a much slower decay than is actually observed. Theoreticians were thereby stimulated to begin their studies of the lunar reflection problem anew.

1 This work was supported in part by the Applied Research Laboratory, Sylvania Electronic Systems, Waltham, Mass.
Senior and Siegel [1959] first proposed what might be termed a "phenomenological" theory in which it was suggested that the lunar echo consisted of a small number (4 or 5) of individual returns, each of which was associated with a single reflecting area. However, other workers (e.g., Hughes [1960]) felt that experimental data obtained with very short pulses failed to support this view. In response to such criticism, Senior and Siegel [1960] later increased their estimate of the number of scattering areas to 25 or 30 , all of which were located within a depth of about $50 \mathrm{~km}$ from the subterrestrial point of the moon. However, a revision of this nature fails to resolve two rather serious objections. First, the theory as it is formulated does not provide a functional formalism which permits quantitative comparison with experimental results obtained using a variety of pulse lengths. Secondly, on the basis of our current knowledge of the moon's surface, it seems quite unlikely that a number of large relatively smooth "spherical surfaces" are concentrated only in the immediate vicinity of the subterrestrial point, while the remainder of the scattering regions over the lunar surface are "craterlike." In any event, the theory of Senior and Siegel has not received wide acceptance and most recent work has used a statistical approach.

The basic premise of the statistical theories is that at any instant of time the amplitude of the lunar echo represents the net effect of scattering from a large number of comparatively extensive reflecting areas randomly oriented and distributed over the surface. Several workers have reported theoretical results in this area, notably Hargreaves [1959], Daniels [1960a, 1960b], Brown [1960], and Hagfors [1961]. These theories need not be reviewed here since a brief summary of each is available in a recent survey paper by Evans [1961].

Section 2 of this paper is devoted to a discussion of lunar scattering mechanisms in which it is proposed that the total echo at any instant of time is the result of reflection from two general types of terrain features. One of these is described by a statistical model which is discussed in some detail in section 3 . 
The radar cross section of the moon is calculated in section 4 . Sections 5 and 6 are devoted to derivations of the temporal distribution of lunar echo power in the case of short pulse transmission. Finally, several comparisons between theory and experiment are given in section 7 .

\section{Discussion of Scattering Mechanisms}

The experimentally obtained echo amplitude distribution appearing in figure 1 leads one to suspect that perhaps more than a single scattering mechanism is responsible for the observed decay. Clearly, the initial part of the return is suggestive of a specular type of scattering process rather than a diffuse type. On the other hand, the subsequent portion of the echo reveals just the opposite characteristic. Thus, it seems appropriate to regard the total signal at each instant of time as the combined effect of two scattering components of different types which, for convenience, will be referred to hereafter as the "quasispecular" and the "diffuse" components, respectively.

The quasi-specular return differs from that which would be observed from a smooth moon in that there exists an extended period of signal decay at times greater than the original pulse length. As pointed out by Evans [1961], the results of experiments using different pulse lengths suggest that during this period of decay the echo amplitude represents the net effect of reflection from a large number of randomly distributed scattering areas. This observation leads quite naturally to the so-called "statistical" model for quasi-specular scattering from the lunar surface.

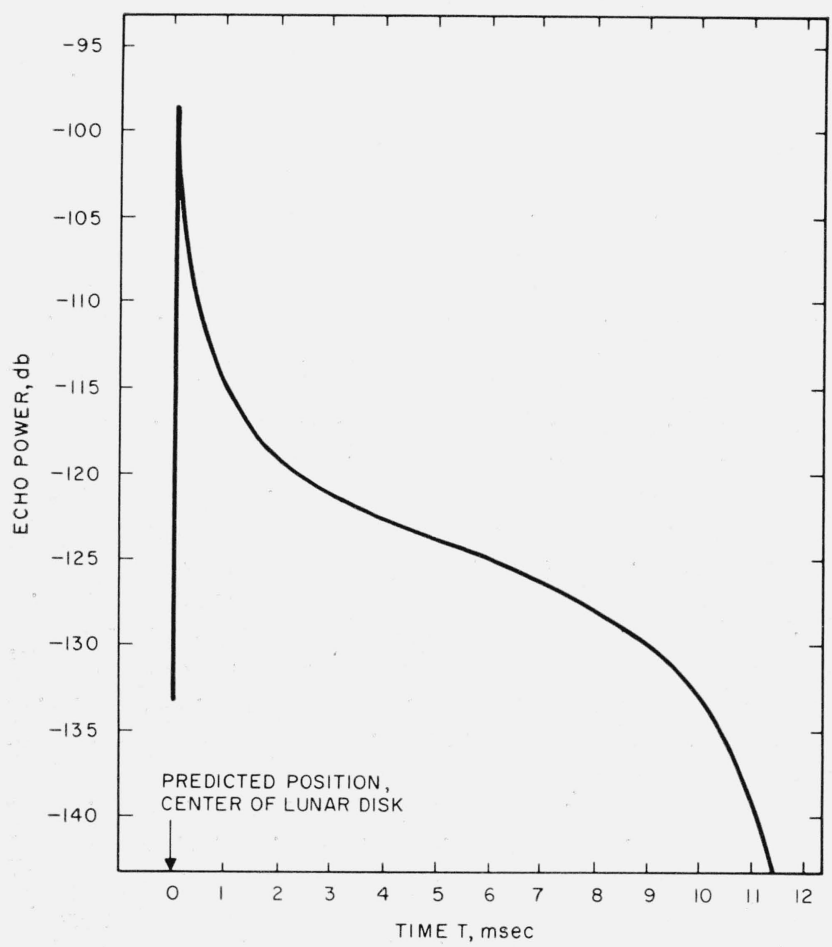

Figure 1. Observed echo power as a function of time for a pulse length" of $100 \mu \mathrm{sec}$.
According to this model, the moon is characterized by random irregularities in surface height $h$. Furthermore, these irregularities are presumed to be large in the sense that their characteristic dimensions are somewhat greater than a few meters and that otherwise the surface is smooth, with the result that incoming radiation is scattered locally in a specular fashion. From the mathematical point of view, it is supposed that $h$ can be treated as a continuous random function of position on the lunar surface and that the statistics of the function $h$ are known, apart from characteristic parameters which are to be determined from experiment.

The experimentally obtained echo decay rate is determined as an average over a large number of returns. Since the rough lunar surface presents a slightly different aspect from one pulse to the next as a result of lunar libration, one might think of each experimentally obtained return (or set of returns, depending upon the prf) as representing the echo obtained from a member of an ensemble of moons characterized by different surface height functions $h$. In an analogous fashion, the average amplitude distribution of the return predicted on the basis of the statistical model is determined by obtaining an ensemble average of the expression for returned power. The average echo distribution will resemble specular reflection much more than diffuse reflection so long as the deviation of the actual surface from the mean is not large and the average surface height-to-separation ratio characteristic of the irregularities is fairly small. On the other hand, it seems quite unlikely that such a scattering mechanism could explain the slow, lowlevel portion of the decay which follows the initial rapid amplitude decrease.

Although the statistical model embodies those physical characteristics of the surface which play a dominant role in backscattering near normal incidence, it is incomplete in the sense that it does not include the existence of small irregular surface features, such as rocks and jagged facets, which will tend to scatter radiation more isotropically. These latter irregularities will contribute relatively little to the amplitude of the signal scattered from the vicinity of the subterrestrial point of the lunar surface. On the other hand, in the case of a short transmitted pulse the echo amplitude at times considerably greater than the pulse length is due to returns from portions of the moon's surface closer to the limb. In this region reflections from small, less directive scattering facets will assume greater importance than quasi-specular reflection from the slow undulatory component of surface roughness. Therefore, it is natural to expect that scattering from these smaller irregular facets will account for the slow decay of echo amplitude which follows the rapid decrease from the peak of the return due to quasi-specular reflection.

In view of the fact that the quasi-specular and diffuse reflection mechanisms operate essentially independently of each other, the total lunar echo power at each instant of time can be considered to 
be the sum of the scattered powers associated with the individual components. Consequently, the power amplitude due to these different scattering mechanisms can be derived independently and the results can subsequently be added to give the composite lunar echo. Since the maximum response from diffuse scattering is 15 to $20 \mathrm{db}$ below that due to quasi-specular reflection, it is apparent that only the latter component need be considered in estimating the total return in the case of continuous wave transmission.

\section{Statistical Model of the Lunar Surface}

The center of the moon is taken to be coincident with the origin of a Cartesian coordinate system as illustrated in figure 2. The transmitter-receiver is located on the positive $x_{3}$-axis at a distance $R$ from the origin.

The surface of the moon is treated as though it were randomly rough. Thus, if the mean surface is assumed to be a sphere of radius $a$, then the local radius $r$ at any point $\mathbf{x}=\left(x_{1}, x_{2}, x_{3}\right)$ on the true surface can be expressed as $r=a+h$, where the local surface height $h$ is a random function of position. In expressions involving the surface configuration it is often convenient to employ spherical coordinates, so that the locus of the point $\mathbf{x}$ can be represented by the parametric system

$$
\begin{aligned}
& x_{1}=r \sin \theta \cos \phi \\
& x_{2}=r \sin \theta \sin \phi \\
& x_{3}=r \cos \theta
\end{aligned}
$$

with $r=a+h(\theta, \phi)$. The local normal vector at $\mathbf{x}$ can be calculated from the expression

$$
\begin{aligned}
\mathbf{n} & =\left[J_{1} \mathbf{e}_{1}+J_{2} \mathbf{e}_{2}+J_{3} \mathbf{e}_{3}\right] /\left[J_{1}^{2}+J_{2}^{2}+J_{3}^{2}\right]^{\frac{1}{2}} \\
& =\left[\frac{\partial \mathbf{x}}{\partial \theta} \times \frac{\partial \mathbf{x}}{\partial \phi}\right] /\left|\frac{\partial \mathbf{x}}{\partial \theta} \times \frac{\partial \mathbf{x}}{\partial \phi}\right|,
\end{aligned}
$$

where $J_{i}(i=1,2,3)$ are the Jacobians of the system $(3-1)$. The $\mathbf{e}_{i}$ are unit vectors in the three mutually orthogonal directions. The local surface area element $d A$ is given by

$$
d A=\left[J_{1}^{2}+J_{2}^{2}+J_{3}^{2}\right]^{\frac{1}{2}} d \theta d \phi .
$$

It is consistent with the hypothesis of locally specular scattering to assume that those surface roughnesses which determine the properties of the quasispecular component are characterized by dimensions which are greater than several radar wavelengths. In the sequel we shall denote by $\sigma$ the standard deviation of the local surface height, and $\delta$ will denote the great circle distance separating two points on the mean lunar surface beyond which local suriace heights are virtually uncorrelated. ${ }^{2}$ In view of the fact that as a radar reflector the near

\footnotetext{
2 The quantity $\delta$ is usually referred to as the "correlation radius."
}

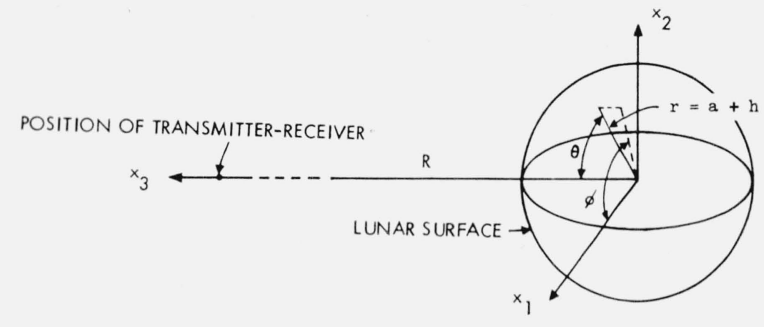

Figure 2. Geometry of the lunar scattering problem.

surface of the moon appears to be much more smooth than rough, it is anticipated that the value of $\sigma / \delta$ will be at least an order of magnitude less than unity. This remark follows from the observation that $\sigma / \delta$ is, in a sense, a measure of the rms surface height-toseparation ratio. Effects such as shadowing and multiple reflections will therefore be of secondary importance near the subterrestrial point of the moon and need not be considered here.

An analysis based on a statistical model of the present sort becomes prohibitively complicated when an attempt is made to take into account explicity the possible electromagnetic properties of the moon's crust. On the other hand, the statistical model proposed here implies a gently undulatory lunar surface, which is smooth over distances of a few radar wavelengths and which scatters radiation specularly in a local sense. This suggests that any given region of the moon's surface must contain areas which are favorably oriented to produce returns if it is to be at all effective in contributing to the back scattered power. This leads to the assumption that the relative importance of any given region in contributing to the returned echo will be more strongly dependent upon the local geometry than upon the electromagnetic parameters of the surface material. In order to determine the influence of local geometry upon the observed echo it has become standard practice in discussions of lunar scattering to assume that the far zone scattered fields can be calculated by taking the total fields at the lunar surface to be the same as though the interior were a perfect dielectric with $\epsilon_{r}=\infty$. The scattered power level in the far zone is later adjusted by a multiplicative correction factor which is usually referred to as an effective power reflection coefficient. The value of this coefficient is determined by calculating the fractional power scattered by a semi-infinite dielectric slab with an effective relative permittivity $\boldsymbol{\epsilon}_{r}$ exposed to a normally incident plane wave. Since the surface areas providing the greatest contribution to the quasi-specular scattering component are those which are perpendicular to the direction of propagation of the incident field, this procedure is a good approximation.

Since a dielectric with $\epsilon_{r}=\infty$ is indistinguishable from a perfect conductor the possibility of internal reflections is implicitly eliminated from consideration. This is consistent with reality since the lunar radius $a$ is so much greater than the skin depth of rocky material at radar wavelengths. 
The section which follows is devoted to the development of an approximate expression for the radar cross section of the moon on the basis of the scattering model just described.

\section{Radar Cross Section of the Moon}

At the conclusion of section 2 it was remarked that the total radar cross section of the moon may be calculated with a fair degree of accuracy by assuming that only the quasi-specular component contributes significantly to the backscattered power. This calculation will be performed using the statistical model for the lunar surface.

In the case of continuous wave transmission we may represent the incident magnetic field as a plane wave propagating in the negative $x_{3}$-direction with a time dependence of the form $\exp (-i \omega t)$ :

$$
\mathbf{H}_{i}=\mathbf{e}_{1} H_{i} \exp \left[-i\left(k x_{3}+\omega t\right)\right] .
$$

The scattered magnetic field can then be calculated from the integral of Stratton and Chu:

$$
\begin{aligned}
\mathbf{H}_{s}=\frac{1}{4 \pi} \int_{A}\left[-i \omega \epsilon_{0}\right. & (\mathbf{n} \times \mathbf{E}) G-\boldsymbol{\nabla} G \\
& \times(\mathbf{n} \times \mathbf{H})+(\mathbf{n} \cdot \mathbf{H}) \nabla G] d A .
\end{aligned}
$$

In this expression, $G$ is the standard Green's function for the three dimensional Helmholtz equation and the electromagnetic vectors in the integrand are the total fields at the surface of the moon. The vector $\mathbf{n}$ is the outward local normal to the true surface at the point $(\theta, \phi)$. Since reflections from the portion of the moon's surface in the shadow region are neglected, the surface $A$ of integration may be taken to be the illuminated hemisphere.

The several assumptions outlined in the previous section permit the use of the so-called "tangent plane approximation." This, together with the idealized electric properties of the surface material, provides us with simple relations for the fields at the surface:

$$
\mathbf{H} \doteq 2 \mathbf{H}_{i}-2\left(\mathbf{H}_{i} \cdot \mathbf{n}\right) \mathbf{n}
$$

and

$$
\mathbf{n} \times \mathbf{E} \doteq 0 .
$$

Following the substitution of (4-3) and (4-4) into the integrand of (4-2) and the specialization of the result to the far zone, the scattered magnetic field can then be written in the form

$$
\begin{aligned}
\mathbf{H}_{s}=-\mathbf{e}_{1}\left[\frac{i k}{2 \pi R}\right] H_{i} & \exp [i(k R \\
& -\omega t)] \int_{A} \exp \left(-i 2 k x_{3}\right) J_{3} d \theta d \phi .
\end{aligned}
$$

The far zone scattered electric field can be written as

$$
\mathbf{E}_{s}=-\zeta_{0} \mathbf{e}_{2} H_{s}
$$

where $\zeta_{0}$ is the characteristic impedance of free space.

The quantity of particular interest is the expected value of the $x_{3}$-component of the Poynting vector $\mathbf{S}$ at the position of the observer. In the case of fields varying harmonically in time, it is customary to suppress the rapid fluctuations of $\mathbf{S}$ by calculating a local time-averaged value. This operation is certainly meaningful for continuous wave transmission when the surface of the scatter is characterized by irregularities considerably greater than a wavelength in dimension. Thus the quantity to be derived is the expected value of

$$
\overline{\mathbf{S}}=\frac{1}{2} \operatorname{Re} \mathbf{E}_{s} \times \mathbf{H}_{s}^{*}
$$

Under the present set of assumptions the Poynting vector at the receiver is directed along the $x_{3}$-axis so that no confusion will arise if $\bar{S}_{x_{3}}$ is written simply as $\bar{S}$.

Equations (4-5) and (4-6) can be combined with $(4-7)$ to give an expression for $\bar{S}$ in terms of the random surface height $h$. The expectation or stochastic average of the power flux is obtained through multiplication of $\bar{S}$ by an appropriate joint probability density function $p\left(h_{1}, h_{2}\right)$ followed by integration over all $h_{1}$ and $h_{2}$. This general method was first presented by Isakovich [1952] and later placed on a more rigorous basis by Hoffman [1955], both of whom treated reflections from a rough conducting plane. After an application of Fubini's Theorem this procedure in the present case leads to the relation ${ }^{3}$

$$
\begin{aligned}
& \mathscr{E}\{\bar{S}\}=\left[\frac{\zeta_{0}}{2} H_{i}^{2}\right]\left[\frac{k}{2 \pi R}\right]^{2} \\
& \operatorname{Re}\left[\int_{A_{1}} \int_{A_{2}} \exp \left[-i 2 k a\left(\cos \theta_{1}-\cos \theta_{2}\right)\right]\right. \\
& \cdot \mathscr{E}\left\{\exp \left[-i 2 k\left(h_{1} \cos \theta_{1}-h_{2} \cos \theta_{2}\right)\right]\right. \\
&\left.\left.J_{31} J_{32}\right\} d \theta_{1} d \theta_{2} d \phi_{1} d \phi_{2}\right] .
\end{aligned}
$$

In order to evaluate this last expression, a number of simplifications must be introduced.

The quantity $J_{3}$ is easily calculated from system $(3-1)$, with the result

$$
J_{3}=r^{2} \sin \theta\left[\cos \theta+\frac{1}{r} \frac{\partial h}{\partial \theta} \sin \theta\right] .
$$

First we observe that $h$ is generally expected to be an extremely small quantity compared with $a$, so that little error is incurred if $r^{2}$ in the first factor is set equal to $a^{2}$. The second term inside the parentheses is nothing more than the product of $\sin \theta$ and the local surface slope in the $\theta$-direction. Current estimates of the rms value of lunar surface slopes

3 Expectations are denoted by $\mathscr{E}\{\}$ to avoid confusion with the symbol $E$ which is reserved for the electric field. 
place this figure at about $10^{\circ}$ or less. Lunar radar data obtained by using very short pulses (see fig. 1) indicate that nearly all of the power returned by the lunar hemisphere is scattered from a region in the vicinity of the subterrestrial point. It would appear that if the estimates of the lunar slopes are reasonably accurate, the second term in parentheses in (4-9) does not assume importance compared with the first except in those regions of the surface which contribute negligibly to the total radar return. This term will henceforth be omitted.

$\mathrm{As}$ a result of the simplifications just outlined, $J_{3}$ no longer involves the random variable $h$ so that $J_{31}$ and $J_{32}$ may be removed from the braces. The expectation which remains is simply the characteristic function appropriate to the joint probability density $p\left(h_{1}, h_{2}\right)$. If we assume this latter function to be the standard Gaussian bivariate distribution with variance $\sigma^{2}$ and correlation function $\rho$, then (4-8) can be expressed in the form

$$
\begin{aligned}
\mathscr{E}\{\bar{S}\}= & {\left[\frac{\zeta_{0}}{2} H_{i}^{2}\right]\left[\frac{k a^{2}}{2 \pi R}\right]^{2} \operatorname{Re}\left[\int _ { A _ { 1 } } \int _ { A _ { 2 } } \operatorname { e x p } \left[-i 2 k a\left(\cos \theta_{1}\right.\right.\right.} \\
- & \left.\left.\cos \theta_{2}\right)\right] \cdot \exp \left[-2 k^{2} \sigma^{2}\left(\cos \theta_{1}\right.\right. \\
- & \left.\left.\cos \theta_{2}\right)^{2}\right] \exp \left[-4 k^{2} \sigma^{2} \cos \theta_{1} \cos \theta_{2}(1-\rho)\right] \\
& \left.\cdot \cos \theta_{1} \cos \theta_{2} \sin \theta_{1} \sin \theta_{2} d \theta_{1} d \theta_{2} d \phi_{1} d \phi_{2}\right] .
\end{aligned}
$$

We first observe that the third exponential factor in the integrand of $(4-10)$ is a rapidly varying function when $\rho$ is near unity. This suggests that we replace $\rho$ by the first few terms of an appropriate series expansion. The correlation function $\rho$ is near unity only when the points $\left(\theta_{1}, \phi_{1}\right)$ and $\left(\theta_{2}, \phi_{2}\right)$ on the mean surface are close to one another. Therefore, throughout that portion of the coordinates domain where the series expansion is no longer valid, the harmonic factor oscillates very rapidly in general, and tends to cancel out residual errors introduced by the expansion.

The functional form of $\rho$ is somewhat arbitrary, but in view of the fact that the mean surface is a sphere, it is natural to assume it to be a function of $\chi / 2$, where $\chi$ is the angle between the lines joining points $\left(\theta_{1}, \phi_{1}\right)$ and $\left(\theta_{2}, \phi_{2}\right)$ with the center of the sphere. A large class of correlation functions, for example, is represented by the relation

$$
\rho=\rho(\sin \chi / 2) .
$$

If we exclude the possibility of a discontinuity in slope at $\chi=0$, we can expand $\rho$ in powers of $\sin \chi / 2$ to obtain

$$
\begin{aligned}
\rho & =1-\frac{1}{2}\left|\rho^{\prime \prime}(0)\right| \sin ^{2} \chi / 2+\ldots \\
& =1-\frac{1}{4}\left|\rho^{\prime \prime}(0)\right|\left[1-\cos \theta_{1} \cos \theta_{2}\right.
\end{aligned}
$$$$
\left.-\sin \theta_{1} \sin \theta_{2} \cos \left(\phi_{1}-\phi_{2}\right)\right]+\ldots
$$

where the prime indicates differentiation with respect to $\sin \chi / 2$.

It is now expedient to take further advantage of the fact that nearly all the power is scattered from the vicinity of the specular reflection point. If we put $x_{i}=\sin \theta_{i}$ in $(4-10)$ then most of the radar signal is returned from the region of small $x_{i}$. Thus we shall set $\cos \theta_{i} \doteq 1-\frac{1}{2} x_{i}^{2}$ throughout the integrand and drop terms which are of an order higher than $x_{i}^{2}$ where appropriate. Further simplification results from the fact that $\frac{1}{2} \rho^{\prime \prime}(0) \mid$ is equal to the product of $\left(2 a^{2} / \sigma^{2}\right)$ and the mean square value of the local surface slope and is much greater than unity except for completely negligible slopes. (It may be noted that in the case of a Gaussian correlation function this product is equal approximately to $4 a^{2} / \delta^{2}$.) Thus we may neglect the terms $k^{2} \sigma^{2} x_{i}^{2}$ as compared with

$\frac{1}{2} k^{2} \sigma^{2}\left|\rho^{\prime \prime}(0)\right| x_{i}^{2},(i=1,2)$. With $\lambda^{2}=k^{2} \sigma^{2}\left|\rho^{\prime \prime}(0)\right|$ and $\nu$ $=2 k a$, the expectation of $\bar{S}$ takes the form

$$
\mathscr{E}\{\bar{S}\} \doteq\left[\frac{\zeta_{0}}{2} H_{i}^{2}\right]\left[\frac{k a^{2}}{4 \pi R}\right]^{2} \operatorname{Re}\{L(k)\}
$$

where

$$
\begin{aligned}
L(k)= & \int_{0}^{1} \int_{0}^{1} \int_{0}^{2 \pi} \int_{0}^{2 \pi} \\
& \exp \left[i \frac{\nu}{2}\left(x_{1}^{2}-x_{2}^{2}\right)\right] \exp \left[-\frac{\lambda^{2}}{2}\left(x_{1}^{2}+x_{2}^{2}\right)\right] \\
& \cdot \exp \left[\lambda^{2} x_{1} x_{2} \cos \left(\phi_{1}-\phi_{2}\right)\right] d x_{1}^{2} d x_{2}^{2} d \phi_{1} d \phi_{2} .
\end{aligned}
$$

If we now put $\mu_{1}=-\frac{1}{2}\left(i \nu-\lambda^{2}\right)$ and $\mu_{2}=\frac{1}{2}\left(i \nu+\lambda^{2}\right)$,

then the result of integration over the azimuthal angles in (4-14) can be written as

$$
L(k)=4 \pi^{2} \int_{0}^{1} \int_{0}^{1} \exp \left[-\left(\mu_{1} x_{1}^{2}+\mu_{2} x_{2}^{2}\right)\right] I_{0}\left(\lambda^{2} x_{1} x_{2}\right) d x_{1}^{2} d x_{2}^{2},
$$

where $I_{0}$ is the zero order modified Bessel function of the first kind. Since $\lambda^{2}$ is a number very much greater than unity, the Bessel function can be expanded asymptotically for $x_{i}$ of the order of unity and greater to show that little error is incurred if the upper limits of integration in (4-15) are set equal to infinity. If $I_{0}$ is then replaced by its series expansion and the summation is removed from the integral signs, then the integrations are easily performed to give as a result

$$
L(k)=\frac{4 \pi^{2}}{\mu_{1} \mu_{2}} \sum_{m=0}^{\infty}\left[\frac{\lambda^{4}}{4 \mu_{1} \mu_{2}}\right]^{m}=\frac{4 \pi^{2}}{k^{2} a^{2}} .
$$


Thus, in the present approximation the expected power flux at the receiver is independent of the precise form of $\left|\rho^{\prime \prime}(0)\right|$. The expected value of $\bar{S}$ is given by

$$
\mathscr{E}\{\bar{S}\} \doteq\left[\frac{\zeta_{0}}{2} H_{i}^{2}\right]\left[\frac{a}{2 R}\right]^{2}
$$

When (4-17) is multiplied by an effective reflection coefficient $\alpha$, standard radar formulas can be used to show that the effective radar cross section of the moon in the present approximation is

$$
\sigma_{s} \doteq \alpha \pi a^{2} .
$$

This is exactly the same result obtained by Grieg, Metzger, and Waer [1948] for a smooth moon; it differs from the result for a perfectly diffuse moon by a factor of $8 / 3$. The difference between the theory just outlined and the smooth moon model is, of course, that the power in each case is scattered from different regions of the surface as we shall see in the next section.

In order to obtain a quantitative comparison of the results just derived with experimental data, it is necessary to assign a reasonable value to the power reflection coefficient $\alpha$. If it is assumed that the lunar surface is composed of material with an effective relative permittivity $\boldsymbol{\epsilon}_{r}$, then the coefficient $\alpha$ can be estimated by calculating the fractional power scattered by a semi-infinite dielectric slab exposed to a normally incident plane wave:

$$
\alpha \doteq\left[\frac{\sqrt{\epsilon_{r}}-1}{\sqrt{\epsilon_{r}}+1}\right]^{2} .
$$

In order to complete the calculation an estimate must be made of the relative permittivity of the lunar surface.

The composition of the lunar crust has been the subject of considerable speculation for a great many years. On the basis of many studies at infrared wavelengths the surface appears to be composed of a granular rocky substratum lying beneath a very thin layer of dust. Thus it appears reasonable to conclude that for our purposes the lunar surface is composed of dust-like granular rock.

Of course, little is known about the precise types of rocky substances to be found in the lunar crust. On the other hand, many well-considered "guesses" have been made in order to provide estimates of the physical constants of the lunar surface. A typical value of the various relative permittivities of such substances is close to 8 . Taking this to be a representative value for lunar surface substances in the solid state, an estimate can be made of the effective permittivity of the material in a dust-like or granular state using formulas such as those developed by DeLoor [1958].

It can thus be shown that the effective relative permittivity of an aggregate of closely packed spherical granules of rock, in vacuo, whose relative permittivity $\epsilon_{1}$ in the solid state is 8 , is equal roughly to
4. Substituting this value into (4-19), the theoretical radar cross section of the moon is approximately

$$
\sigma_{s} \doteq 0.11 \pi a^{2} \text {. }
$$

Ample data exist to check this result against experimental findings. Senior and Siegel [1959], for example, present a table of several measured values of $\sigma_{s} / \pi a^{2}$ over a considerable frequency range. In a recent summary paper on the problem of lunar radar reflections Evans [1961] contributes further values at higher frequencies. As pointed out by Evans, the radar cross section of the moon shows no systematic variation with wavelength over the interval $3 \mathrm{~m}$ to $3 \mathrm{~cm}$ and the average value obtained experimentally is

$$
\sigma_{s} \doteq(0.081 \pm 0.005) \pi a^{2}
$$

which agrees well with the result in (4-20).

\section{Pulse Transmission: The Quasi-Specular Component}

Lunar radar reflection experiments in which short pulses were transmitted provided somewhat more insight into the nature of the moon as a scatterer of electromagnetic radiation than could be gained from continuous wave transmission. In the case of very short transmitted pulses the temporal distribution of echo power can be interpreted as a measure of effectiveness of various parts of the moon's surface in scattering radiation back to the Earth. As already mentioned, the average power received at any instant can be thought of as the sum of the quasispecular and the diffuse components. The quasispecular component dominates that part of the return which originates in the vicinity of the subterrestrial point on the moon. The discussion which follows is devoted to the derivation of expressions for power received from quasi-specular scattering as a function of time. The scattering model is the same as that described in section 3 .

In the case of transmission of a rectangular pulse of duration $\tau$ the incident magnetic field can be expressed in the form

$$
\begin{aligned}
\mathbf{H}^{i}= & \mathbf{e}_{1} H_{i} \exp \left[-i\left(k x_{3}+\omega t\right)\right]\left[U\left(c t+x_{3}\right)-U\left(c t-c \tau+x_{3}\right)\right] \\
= & \mathbf{e}_{1} H_{i} \frac{1}{2 \pi i} \int_{-\infty}^{\infty} \exp \left[i\left(c t+x_{3}\right) k_{1}\right][1 \\
& \left.\quad-\exp \left[-i\left(k+k_{1}\right) c \tau\right]\right]\left[\frac{1}{k_{1}+k}\right] d k_{1}, \quad(5-1)
\end{aligned}
$$

where $U$ denotes the Heaviside step function. It should be remarked that the path of integration in the second expression passes below the pole on the real axis. If $\mathbf{H}_{i}(k)$ denotes the incident wave in the case of continuous wave transmission, apart from the time factor $\exp (-i \omega t)$, then an alternative expression to $(5-1)$ is 


$$
\begin{aligned}
\mathbf{H}^{i}=\frac{1}{2 \pi i} \int_{-\infty}^{\infty} \exp & \left(i k_{1} c t\right)\left[1-\exp \left[-i\left(k_{1}\right.\right.\right. \\
& +k) c \tau]] \mathbf{H}_{i}\left(-k_{1}\right)\left[\frac{1}{k_{1}+k}\right] d k_{1} .
\end{aligned}
$$

Thus if $\mathbf{H}_{s}(k)$ denotes the scattered field (4-5) without the factor $\exp (-i \omega t)$, then the scattered magnetic field in the case of pulse transmission is just

$$
\begin{array}{r}
\mathbf{H}^{s}=\frac{1}{2 \pi i} \int_{-\infty}^{\infty} \exp \left(i k_{1} c t\right)\left[1-\exp \left[-i\left(k_{1}\right.\right.\right. \\
+k) c \tau]] \mathbf{H}_{s}\left(-k_{1}\right)\left[\frac{1}{k_{1}+k}\right] d k_{1} .
\end{array}
$$

As in the previous analysis, the scattered electric field can be obtained from the relation

$$
\mathbf{E}^{s}=-\zeta_{0} \mathbf{e}_{2} H^{s}
$$

These expressions will be used subsequently to calculate the expected value of received power as a function of time.

Once again, the quantity of interest is the expected value of the $x_{3}$-component of the Poynting vector $\mathbf{S}$ at the position of the observer. In the case of continuous wave transmission, the rapid fluctuations of $\mathbf{S}$ were suppressed by calculating a local timeaveraged value. In the case of pulse reflection, the same time average must be interpreted as a local "adiabatic" average in which the time dependent coefficients of the harmonic factors are regarded as slowly varying over several periods of the carrier wave. In the case of gross roughness, this is a good approximation except possibly in a few very narrow intervals in the time domain (such as the expected position of the leading edge of the return). Since experimental data are uncertain in these regions, the precise time average at these points deserves no special consideration. Thus for pulse transmission the quantity to be derived is the stochastic average of

$$
\overline{\mathbf{S}}=\frac{1}{2} \operatorname{Re} \mathbf{E}^{s} \times \mathbf{H}^{* *}
$$

Just as in the case of CW transmission the present set of assumptions leads to a Poynting vector in the $x_{3}$-direction at the receiver so that $\bar{S}_{x_{3}}$ will again be written simply as $\bar{S}$.

It is convenient to shift the origin of time so that the "average" leading edge of the scattered pulse arrives at time $T=0$; i.e., put $c t=c T+R-2 a$ in $(5-3)$ and $(5-4)$. Some degree of brevity is achieved by setting

$$
F\left(k_{i}\right)=\left[1-\exp \left[-i\left(k_{i}+k\right) c \tau\right]\right]\left[\frac{k_{i}}{k_{i}+k}\right] .
$$

Here, $\tau$ is the duration of the transmitted pulse and $k_{i}$ is a transform variable. The expected value of $\bar{S}$ can then be written in the form

$$
\begin{aligned}
& \mathscr{E}\{\bar{S}\}=\left[\frac{\zeta_{0}}{2} H_{i}^{2}\right] \frac{1}{\left(4 \pi^{2} R\right)^{2}} \operatorname{Re}\left[\int_{-\infty}^{\infty} \int_{-\infty}^{\infty} \int_{A_{1}} \int_{A_{2}}\right. \\
& \cdot \exp \left[i(c T-2 a) k_{1}-i(c T-2 a) k_{2}\right] \cdot F\left(k_{1}\right) F^{*}\left(k_{2}\right) \\
& \cdot \exp \left[i 2 k_{1} a \cos \theta_{1}-i 2 k_{2} a \cos \theta_{2}\right] \\
& \cdot \mathscr{E}\left\{\exp \left[i 2 k_{1} h_{1} \cos \theta_{1}-i 2 k_{2} h_{2} \cos \theta_{2}\right]\right. \\
&\left.\left.J_{31} J_{32}\right\} d \theta_{1} d \theta_{2} d \phi_{1} d \phi_{2} d k_{1} d k_{2}\right]
\end{aligned}
$$

It is important to note that the path of integration in the second transform (involving $k_{2}$ ) passes over the pole on the real axis since we have taken the complex conjugate of the expression for $\mathbf{H}_{s}$.

In the remainder of the discussion we shall confine our attention to pulses of very short duration $(\tau<<<2 a / c)$ and to comparatively small values of time $T$. With these restrictions, the same kind of approximations used in the CW analysis will apply to the present situation. For example, when $T$ is very small compared with $2 a / c$, that portion of the lunar hemisphere contributing to the scattered power is characterized by small $x_{i}=\sin \theta_{i}$. Moreover, the receiver bandwidths employed in lunar scattering experiments are such that only those frequencies within a few tens of megacycles of the carrier frequency are of significance from the equipment standpoint. Although the variation of $k_{1}$ and $k_{2}$ in the transforms corresponds to all frequencies, in so far as the geometric approximations are concerned, products such as $k_{i} k_{j} \sigma^{2}$ can be treated as being of the order of $k^{2} \sigma^{2}$. Hence, apart from a few obvious modifications, the simplification of the integrand in $(5-7)$ proceeds in the same fashion as before, giving as a result

$$
\begin{array}{r}
\mathscr{E}\{\bar{S}\}=\left[\frac{\zeta_{0}}{2} H_{i}^{2}\right]\left[\frac{a^{2}}{8 \pi^{2} R}\right]^{2} \operatorname{Re}\left[\int _ { - \infty } ^ { \infty } \int _ { - \infty } ^ { \infty } \operatorname { e x p } \left[i c T k_{1}\right.\right. \\
\left.-i c T k_{2}\right] \exp \left[-2 \sigma^{2}\left(k_{1}-k_{2}\right)^{2}\right] \\
\left.\cdot F\left(k_{1}\right) F^{*}\left(k_{2}\right) L\left(k_{1}, k_{2}\right) d k_{1} d k_{2}\right]
\end{array}
$$

where

$$
\begin{gathered}
L\left(k_{1}, k_{2}\right)=\int_{0}^{1} \int_{0}^{1} \int_{0}^{2 \pi} \int_{0}^{2 \pi} \exp \left[-\frac{1}{2}\left(i \nu_{1}+\lambda^{2}\right) x_{1}^{2}\right] \\
\cdot \exp \left[\frac{1}{2}\left(i \nu_{2}-\lambda^{2}\right) x_{2}^{2}\right] \\
\cdot \exp \left[\lambda^{2} x_{1} x_{2} \cos \left(\phi_{1}-\phi_{2}\right)\right] d x_{1}^{2} d x_{2}^{2} d \phi_{1} d \phi_{2} .
\end{gathered}
$$

In this last expression, $\lambda^{2}=k_{1} k_{2} \sigma^{2}\left|\rho^{\prime \prime}(0)\right|, \quad \nu_{1}=2 k_{1} a$, and $\nu_{2}=2 k_{2} a$.

The integration in (5-9) is performed in much the same manner as that in $(4-14)$. The result can be expressed in the form

$$
L\left(k_{1}, k_{2}\right)=\frac{4 \pi^{2}}{k_{1} k_{2} a^{2}}\left[1+i \frac{1}{\beta}\left(k_{1}-k_{2}\right)\right]^{-1},
$$

where $\beta=2 a /\left|\rho^{\prime \prime}(0)\right| \sigma^{2}$.

The calculation of the resulting transforms in $(5-8)$ is somewhat tedious and will not be reproduced 
here. The final expression for the expectation of $\bar{S}$, under current restrictions, can be conveniently written as

$$
\begin{aligned}
& \mathscr{E}\{\overline{\boldsymbol{S}}\}=\left[\frac{\zeta_{0}}{2} H_{i}^{2}\right]\left[\frac{a}{2 R}\right]^{2} {\left[\frac{1}{2} \exp \left(2 \beta^{2} \sigma^{2}\right)\right.} \\
&\cdot[J(c T)-J(c T-c \tau)]],
\end{aligned}
$$

where

$$
\begin{aligned}
J(u)= & \exp \left(-2 \beta^{2} \sigma^{2}\right)[1+\operatorname{erf}(u / 2 \sqrt{2} \sigma)] \\
& -\exp (-\beta u)[1+\operatorname{erf}(u / 2 \sqrt{2} \sigma-\sqrt{2} \beta \sigma)] .
\end{aligned}
$$

Although $(5-11)$ is a relatively uncomplicated relation and well suited to rapid calculations by an electronic digital computer, more insight into the gross functional dependence of $\mathscr{E}\{\bar{S}\}$ on time is provided by further simplification. We first observe that the product $\beta \sigma$ is a number very much less than unity (except for completely negligible lunar surface slopes). Furthermore, on the basis of several calculations described elsewhere [Winter, 1961], it has been shown that the principal role of the error functions appearing in $(5-12)$ is to smooth out discontinuities in slope which would otherwise appear if $\sigma$ were allowed to approach $0+$ in their arguments. ${ }^{4}$ Therefore, replacing $\beta \sigma$ by zero and noting that

$$
\lim _{\sigma \rightarrow 0+} \frac{1}{2}[1+\operatorname{erf}(u / 2 \sqrt{2} \sigma)]=U(u),
$$

it can be shown that an approximate relation for the expected value of $\bar{S}$ is

$$
\begin{aligned}
\mathscr{E}\{\bar{S}\} & \doteq\left[\frac{\zeta_{0}}{2} H_{i}^{2}\right]\left[\frac{a}{2 R}\right]^{2} \\
& \begin{cases}0 \quad & \quad T<0 \\
1-\exp (-\beta c T) & 0<T<\tau \\
{[\exp (\beta c \tau)-1] \exp (-\beta c T) ;} & \tau<T<<2 a / c .\end{cases}
\end{aligned}
$$

Thus for values of $T$ greater than the pulse duration the decay of echo power is approximately exponential, with a "time constant" equal to $1 / \beta c$.

\section{Pulse Transmission: The Diffuse Component}

The quasi-specular component of the lunar echo is due to scattering from that portion of the lunar terrain which appears to be locally smooth and whose irregularities are large compared with a wavelength. In the case of short pulse transmission this component will decrease in importance very rapidly as the "illuminated zone" moves back toward the limb from the subterrestrial point. At the same time scattering from smaller, rougher surface features

${ }^{4}$ This is equivalent to letting $\sigma \rightarrow 0+$ in the factor exp $\left[-2 \sigma^{2}\left(k_{1}-k_{2}\right)^{2}\right]$ appearing in $(5-8)$. (such as rocks and jagged facets) will increase in relative importance since these facets will tend to scatter radiation in a more isotropic fashion. Since the detailed structure of such features is not known, a semiempirical approach must be employed.

We begin by assuming that echo power associated with the diffuse component is due to scattering from a great number of small scatterers which are randomly distributed over the surface of the moon. Suppose that the $j$ th scatterer at $\left(\theta_{j}, \phi_{j}\right)$ is situated within the small area element $\Delta A_{j}$. The average total power incident upon $\Delta A_{j}$ is approximately

$$
\bar{P}_{\text {inc }}=\left[\frac{\zeta_{0}}{2} H_{i}^{2}\right] f\left(R_{j}\right) \cos \theta_{j} \Delta A_{j},
$$

where $R_{i}$ is the range from the transmitter-receiver to the scatterer and the factor $f\left(R_{j}\right)$ is such that if $g\left[c T-\left(R_{j}+a-R\right)\right]$ is the instantaneous distribution of transmitted power in range, then

$$
f\left(R_{j}\right)=g\left[c T-2\left(R_{j}+a-R\right)\right] .
$$

Thus for a rectangular pulse of duration $\tau$ arriving at the subterrestrial point on the moon at time $T=0$, the factor $g$ takes the form

$$
\begin{aligned}
g\left[c T-\left(R_{j}+a-R\right)\right] & =U\left[c T-\left(R_{j}+a-R\right)\right] \\
& -U\left[c T-c \tau-\left(R_{j}+a-R\right)\right] .
\end{aligned}
$$

Let $\bar{\sigma}_{k}\left(\theta_{j}, \phi_{j}\right)$ denote the radar cross section per unit area of the $j$ th scatterer. The power flux at the receiver due to scattering from this facet is then

$$
\bar{S}_{j}=\bar{P}_{\text {inc }}\left[\frac{1}{4 \pi R_{j}^{2}}\right] \bar{\sigma}_{k}\left(\theta_{j}, \phi_{j}\right) \sec \theta_{j} .
$$

Since it has been assumed that the scatterers are distributed over the lunar surface in a random fashion, the relative phases of waves reflected from them will be randomly distributed and uncorrelated and the power flux at the receiver at any instant of time is simply the sum of the individual contributions. Combining $(6-1),(6-2)$, and $(6-3)$ with (6-4) the received power flux can be written as

$$
\begin{array}{r}
\mathscr{E}\{\bar{S}\}=\left[\frac{\zeta_{0}}{2} H_{i}^{2}\right] \sum_{j=1}^{N}\left\{U\left[c T-2\left(R_{j}+a-R\right)\right]\right. \\
\left.-U\left[c T-c \tau-2\left(R_{j}+a-R\right)\right]\right\} \\
\cdot\left[\frac{1}{4 \pi R_{j}^{2}}\right] \bar{\sigma}_{k}\left(\theta_{j}, \phi_{j}\right) \Delta A_{j},
\end{array}
$$

where $N$ is the number of scatterers.

Since $\bar{\sigma}_{k}\left(\theta_{j}, \phi_{j}\right)$ is not known, it is convenient to treat it as a random variable with a probability density function $p\left[\bar{\sigma}_{k}\left(\theta_{j}, \phi_{j}\right)\right]$. The expected value of $\bar{S}$ may then be defined as

$$
\mathscr{E}\{\bar{S}\}=\sum_{k=1}^{M} \bar{S}\left(\bar{\sigma}_{k}\right) p\left[\bar{\sigma}_{k}\left(\theta_{j}, \phi_{j}\right)\right] \Delta \bar{\sigma}_{k} .
$$


Equation (6-6்) can be combined with (6-5) to give an explicit expression for $\mathscr{E}\{\bar{S}\}$. If we allow the number of scatterers to approach infinity and let all $\Delta \bar{\sigma}_{k}$ to go to zero (thus forcing $M$ to approach infinity) the summations become integrations over $\theta, \phi$, and $\bar{\sigma}$. The range $R_{j}$ is then given by $R-a \cos \theta$. The factor $1 / 4 \pi R_{j}^{2}$ may be replaced by $1 / 4 \pi R^{2}$ and subsequently be removed from the integral. With these modifications, the expectation of $\bar{S}$ takes the form

$$
\begin{array}{r}
\mathscr{E}\{\bar{S}\}=\left[\frac{\zeta_{0}}{2} H_{i}^{2}\right]\left[\frac{a^{2}}{4 \pi R^{2}}\right] \int_{0}^{\infty} \int_{0}^{\pi / 2} \int_{0}^{2 \pi} \\
{[U(c T-2 a+2 a \cos \theta)-U(c T-c \tau-2 a+2 a \cos \theta)]} \\
\cdot \bar{\sigma}(\theta, \phi) p[\bar{\sigma}(\theta, \phi)] \sin \theta d \theta d \phi d \bar{\sigma} .
\end{array}
$$

The integral over $\bar{\sigma}$ is nothing more than the mean $\bar{\sigma}_{0}(\theta, \phi)$ of the random variable $\bar{\sigma}(\theta, \phi)$. Thus, in order to complete the calculation some additional statements must be made with regard to $\bar{\sigma}_{0}(\theta, \phi)$.

As was stated earlier, in the absence of any detailed information concerning the structure of the less directive scattering facets, it is impossible to assign a precise functional form to $\bar{\sigma}_{0}(\theta, \phi)$. On the other hand, since the facets are presumably distributed over the surface in a random fashion, there is no reason to retain an azimuthal dependence explicitly. This dependence could, in fact, be determined (in theory) only if the configuration of the facets were speciified together with their orientation with respect to the direction of the incident field vectors. In suppressing the azimuthal dependence, the parameter $\bar{\sigma}_{0}(\theta)$ can be interpreted as the value of $\bar{\sigma}_{0}(\theta, \phi)$ averaged over the angle $\phi$.

The experimentally determined behavior of the diffuse component (e.g., see ifg. 1) suggests that $\bar{\sigma}_{0}(\theta)$ may be represented in the form

$$
\bar{\sigma}_{0}(\theta)=\sigma_{0}(\cos \theta)^{n},
$$

where $n$ is of the order of magnitude of unity or more. If the surface facets reradiated energy isotropically, and shadowing were unimportant, then $n$ would be identically equal to unity. On the other hand, we shall see in section 7 that Pettengill's experiments suggest the net effect of backscattering from these facets is more adequately described by Lambert's Law which implies that $n$ is closer to two. It should not be concluded from this, however, that the features of the lunar surface producing the diffuse echo component scatter radiation in the same sense that a "gray body" is diffuse at infrared wavelengths. In fact, if further experimentation verifies this law, and if the present model is correct, the diffuse component may be interpreted as scattering from certain preferred types of connigurations, such as spikes or other sharply pointed facets. Although the following is not particularly appropriate to the physical situation at hand, Ament [1956] has shown, by way of example, that Lambert's Law is suggested in backscattering from the coplanar ends of parallel but randomly situated semi-infinite thin wires.
At any rate, when (6-8) is substituted into (6-7), the scattered power flux associated with the diffuse component can be written as

$$
\begin{aligned}
& \mathscr{E}\{\bar{S}\}=\left[\frac{\zeta_{0}}{2} H_{i}^{2}\right]\left[\frac{a}{2 R}\right]^{2} \frac{2 \sigma_{0}}{n+1} \\
& \begin{cases}0 & ; \quad T<0 \\
1-\left[1-\frac{c T}{2 a}\right]^{n+1} & ; \quad 0<T<\tau \\
{\left[1-\frac{c(T-\tau)}{2 a}\right]^{n+1}-\left[1-\frac{c T}{2 a}\right]^{n+1} ;} & \tau<T<\frac{2 a}{c}\end{cases}
\end{aligned}
$$

over the time interval of interest here. The total lunar echo power at any instant of time $T$ is the sum of (6-9) and the corresponding expression for $\mathscr{E}\{\bar{S}\}$ associated with quasi-specular reflection. The value of $\sigma_{0}$ is, of course, determined from experimental data.

\section{Comparison of Total Theoretical Signal With Experiment}

Although experimental attempts to obtain lunar echoes using short pulse transmission have been in progress throughout the past decade, detailed results of such studies have appeared in the literature only within three or four years of this writing. Inadequacies in existing equipment, coupled with large transmission losses, still make it difficult to attach a great amount of accuracy to any collection of lunar radar observations. Therefore, it is not unadvisable to regard the experimental results available at the present time with some reservation. On the other hand, a number of experiments, with various carrier frequencies and pulse lengths, are sufficiently reliable so that a satisfactory degree of accuracy may be attributed to the measurements. Amongst these data are those reported by Trexler [1958], Hughes [1960], and Pettengill [1960, 1961]. Theoretical results derived in the present investigation are compared with the aforementioned experimental results and others in the discussion below.

Among the most accurate lunar radar data available are those of Pettengill [1961]. The equipment employed in this experiment was the Millstone Radar of Massachusetts Institute of Technology's Lincoln Laboratory. Using a transmitted carrier frequency of $440 \mathrm{Mc} / \mathrm{s}$ and a pulse length of 100 $\mu$ sec, Pettengill was able to obtain an average temporal distribution of echo power throughout a time interval corresponding to the total radar "depth" of the moon (11.6 msec). Figure 1 presents the results as reported by Pettengill. The precise behavior of the initial rise of the signal from the vicinity of $T=0$ is not well known so that only the period of signal decay will be compared with theory.

The task of fitting the theoretical results to the experimental curve involves the solution of a three 
parameter problem since the total signal is given by the sum of (5-14) and (6-9) which together involve the constants $\beta, \sigma_{0}$, and $n$. Instead of assigning values to the constant $\beta$ we shall refer to

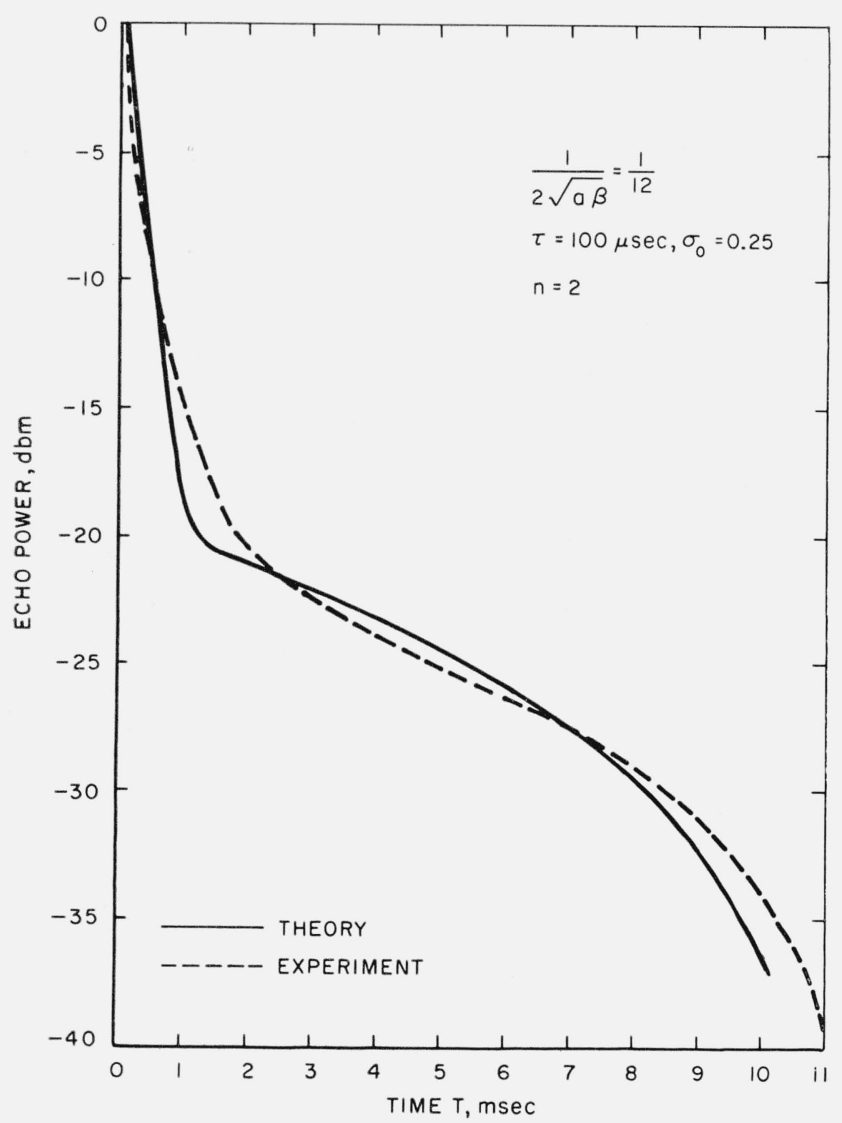

Figure 3. Comparison between theory and experiment over an entire echo.

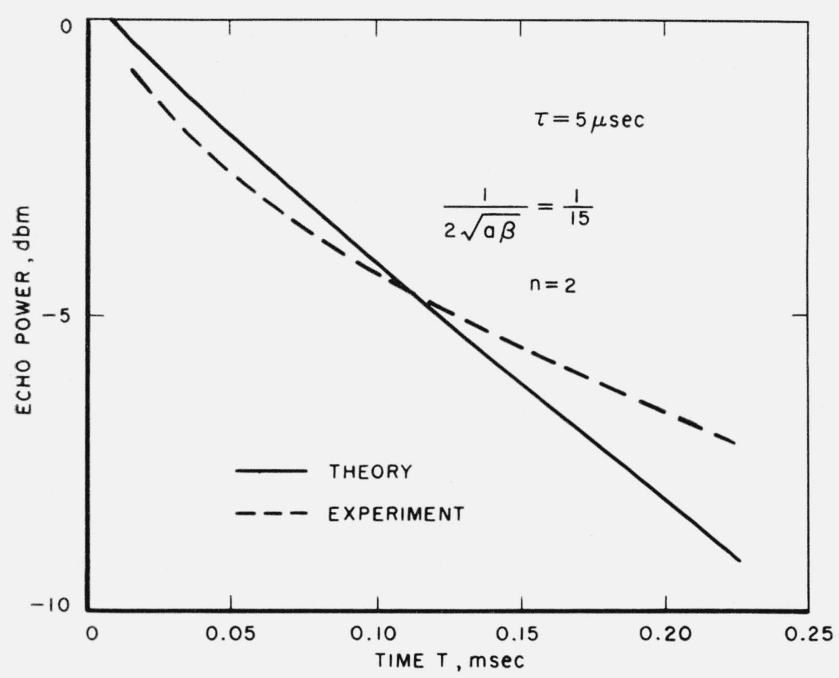

Figure 4. Comparison of theoretical results with the initial portion of the echo decay for a very short pulse. the combination $1 / 2 \sqrt{a \beta}$. From the definition of $\beta$ and the remarks in section 4 with regard to the relation between $\left|\rho^{\prime \prime}(0)\right|$ and the mean square lunar surface slope, it can be seen that

$$
\frac{1}{2 \sqrt{a \beta}}=\left[\frac{\sigma}{2 a}\right]\left[\frac{1}{2}\left|\rho^{\prime \prime}(0)\right|\right]^{\frac{1}{2}}=\frac{1}{\sqrt{2}}\left[\mathscr{E}\left\{\left[\frac{1}{a} \frac{d h}{d x}\right]^{2}\right\}\right]^{\frac{1}{2}} .
$$

In the special case of a Gaussian correlation function, the various expressions in $(7-1)$ are equal simply to $\sigma / \delta$ (for $\delta<<a$ ).

On the basis of several calculations it was concluded that the best overall agreement between the theoretical curve and the experimental one is achieved by using parameter values of the order of

$$
\begin{aligned}
& 1 / 15 \leq 1 / 2 \sqrt{a \beta} \leq 1 / 12 \\
& 1 / 4 \leq \sigma_{0} \leq 1 / 3 \\
& 3 / 2 \leq n \leq 2 .
\end{aligned}
$$

The graph corresponding to the set $(1 / 12,0.25,2)$ is shown together with the experimental decay rate in iigure 3 .

Of course, comparison of the theory with a single experiment is inconclusive. The general usefulness of the present formulation will be demonstrated if it can be shown that comparisons with other experimental results lead to values for $1 / 2 \sqrt{a \beta}, \sigma_{0}$, and $n$ which are close to those indicated in $(7-2)$.

Recent work reported by Hughes [1960] provides us with results of an experiment performed with equipment parameters which are considerably different from those of the Millstone Radar. Using a $5 \mu$ sec pulse and a carrier frequency of $3000 \mathrm{Mc} / \mathrm{s}$, Hughes observed a lunar echo decay which seemed to be fairly well represented by the function

$$
\bar{S}=\bar{S}_{0} \exp \left(-10 \theta_{f}\right) ; \quad 3^{\circ} \leq \theta_{f} \leq 14^{\circ}
$$

where $\theta_{f}=\operatorname{arc} \cos (1-c T / 2 a)$. This expression is plotted in figure 4 . The theoretical result corresponding to the set of parameter values $(1 / 2 \sqrt{a \beta}$, $\left.\sigma_{0}, n\right)=(1 / 15,0.25,2)$ appears to give a fairly good overall fit to the data.

Inspection of figures 3 and 4 reveals a systematic difference in slope between the theoretical and experimental results even though the parameter values fall within the ranges indicated in (7-2). Similar disparity in slope was encountered by Hagfors [1961] who assumed a Gaussian correlation function to derive an "apparent delay spectrum," which can be interpreted as a measure of the relative effectiveness of various portions of the lunar surface in returning a radar signal. Hagfors remarks that the discrepancy in slope might be removed by a different choice for the correlation function $\rho$. Evans [1961] concurs with this view. Another possible explanation is suggested by an inspection 
of lunar photographs or charts of lunar topography which reveals that the subterrestrial point lies within a comparatively smooth flat region which gradually blends into somewhat rougher terrain around its borders. Thus it may be that the effective rms value of local lunar surface slope actually increases slightly with increasing $\theta$ in this general region. In this event the predicted rate of decay of echo power with time would slowly decrease with increasing $T$ as seems to be observed in practice.

Theories based on the statistical model of the moon's surface have been criticized in the past for their inability to account for the so-called modulation loss phenomenon. The term "modulation loss" refers to the apparent decrease in the peak power of the lunar echo as the transmitted pulse duration is diminished. The present formulation, on the other hand, provides an explicit expression for the modulation loss for comparison with observed data. Although the appropriate experimental results are sparse and somewhat uncertain, the most reliable are probably those communicated to Senior and Siegel [1960] by Youmans. According to these data the peak power returned from a $200 \mu$ sec pulse is about $1 \mathrm{db}$ below the value for continuous wave transmission. The corresponding value for a 30 $\mu$ sec pulse is $8.5 \mathrm{db}$, for a $10 \mu$ sec pulse it is $17 \mathrm{db}$, and for a $2 \mu$ sec pulse the loss appears to be $22 \pm 2$ db. An approximate theoretical expression for the modulation loss as a function of pulse length is obtained from a combination of (4-17) and (5-14):

$$
\text { Loss }=1-\exp (-\beta c \tau) .
$$

Figure 5 presents a plot of the modulation loss predicted from $(7-4)$ with $1 / 2 \sqrt{a \beta}=1 / 12$ together with the experimental results described above. The agreement is seen to be quite satisfactory.

Another check of the foregoing results was performed using the data obtained by Trexler [1958] who employed a pulse length of $12 \mu$ sec and a carrier frequency of $198 \mathrm{Mc} / \mathrm{s}$. Trexler presents a plot of the fraction of total echo energy received at time $T$. With (5-14) it is a simple matter to obtain an expression for this fraction as a function of time. Denoting this quantity by $Q$, for $T \geq \tau$, we have

$$
Q \doteq 1-(\beta c \tau)^{-1}[\exp (\beta c \tau)-1] \exp (-\beta c T) .
$$

Figure 6 displays curves of $\log _{e}(1-Q)$ as a function of $T$ for $\tau=12 \mu$ sec. The circled points were calculated from Trexler's plot of experimental results. It would appear that the best fit to the radar data is obtained by using the ratio $1 / 2 \sqrt{a \beta}=1 / 17.5$, a value slightly lower than indicated in $(7-2)$. The theoretical curve corresponding to $1 / 2 \sqrt{a \beta}=1 / 15$ is shown for comparison.

A check of the parameters $\sigma_{0}$ and $n$ is not readily available since only one or two lunar radar equipments have sufficient sensitivity to detect the low level decay associated with the tail of the echo. However, Pettengill [1960] has also reported the results of experiments with the Millstone Radar in

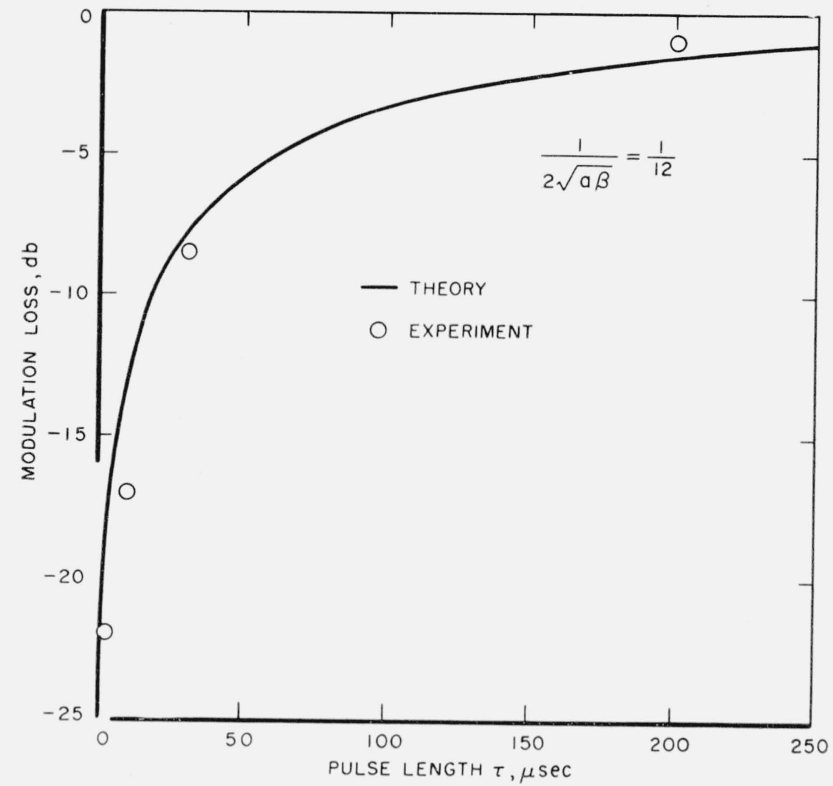

Figure 5. Comparison between theoretical and experimental modulation loss.

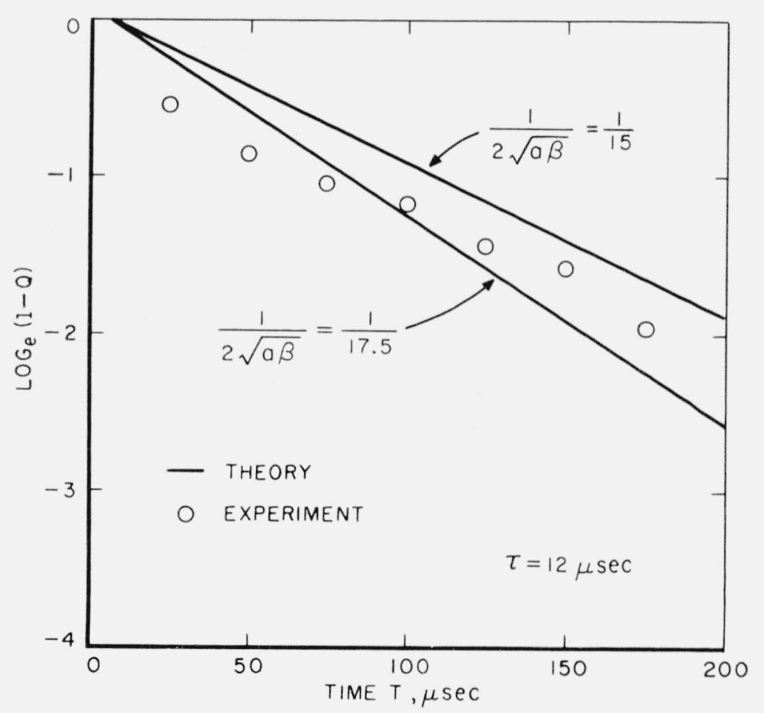

Figure 6. Comparison between theoretical and observed fraction of echo energy returned at time $T$.

which $500 \mu$ sec pulses were used. That portion of the experimental results corresponding to the diffuse component is illustrated in figure 7 together with the theoretical curve corresponding to $\left(1 / 2 \sqrt{a \beta}, \sigma_{0}\right.$, $n)=(1 / 12,0.3,2)$. Again the parameters fall within the ranges indicated by $(7-2)$ and overall agreement is quite good. Comparison with portions of the experimental curve corresponding to times less than $1 \mathrm{msec}$ is not possible because the sampling interval employed in the experiment $(500 \mu \mathrm{sec})$ is so great as to obscure the details of the rise and decay of the echo in this region. 


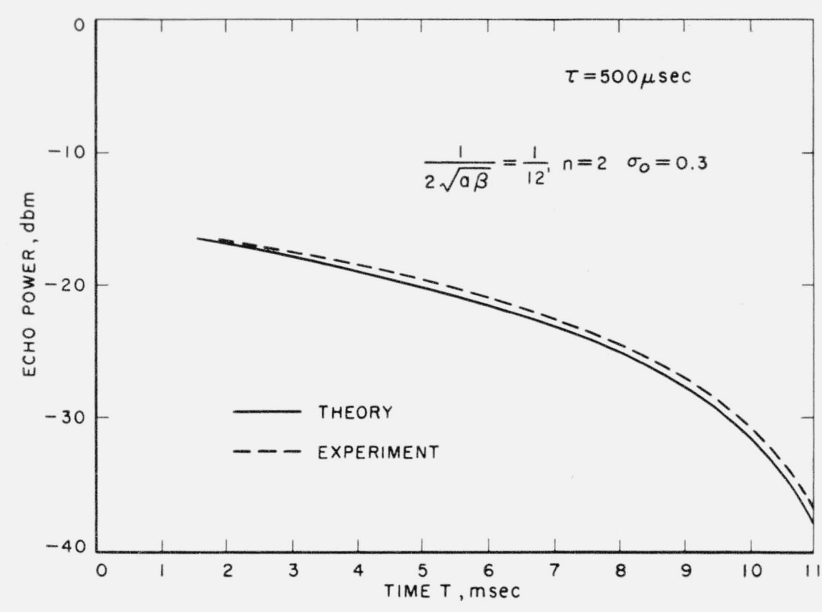

Figure 7. Comparison of theoretical and experimental results for the diffuse component.

The author expresses his thanks to Prof. R. W. P. King and to Drs. J. E. Storer and D. W. Tufts for several helpful discussions of this problem.

\section{References}

Ament, W. S., Forward- and back-scattering from certain rough surfaces, IRE Trans. AP-4, 369 (1956).

Brown, W. E., Jr., A lunar and planetary echo theory, J. Geophys. Research 65, 3087 (1960).

Daniels, F. B., The angular power spectrum of lunar radio echoes, USASRDL Tech. Rep. 2129 (1960a).

Daniels, F. B., A theory of radar reflections from the moon, USASRDL Tech. Rep. 2163 (1960b).
DeLoor, G. P., Thesis, Leiden (1956), as reported by L. Hartshorn and J. A. Saxton, Dispersion and absorption of waves, Handbuch der Physik XVI (ed. S. Flügge) (Springer-Verlag, 1958).

Evans, J. V., The scattering properties of the lunar surface at radio wavelengths, Lincoln Lab. Rep. 3G-0004 (1961).

Grieg, D. D., S. Metzger, and R. Waer, Considerations of moon-relay communication, Proc. IRE 36, 652 (1948).

Hagsfors, T., Some properties of radio waves reflected from the moon and their relation to the lunar surface, J. Geophys. Research 66, 777 (1961)

Hargreaves, Radio observation of the lunar surface, Proc. Phys. Soc. B 73, 536 (1959).

Hoffman, W. C., Scattering of electromagnetic waves from a random surface, Quart. Appl. Math. XIII, 291 (1955).

Hughes, V. A., Roughness of the moon as a radar reflector, Nature 186, 873 (1960).

Isakovich, M. A., The scattering of waves from a statistically rough surface, Zhur. Eksp. i Teoret. Fiz. 23, 305 (1952).

Leadabrand, R. L., R. B. Dyce, A. Fredriksen, R. I. Presnell, and J. C. Schlobohm, Radio frequency scattering from the surface of the moon, Proc. IRE 48, 932 (1960).

Pettengill, G. H., Measurement of lunar reflectivity using the Millstone Radar, Proc. IRE 48, 933 (1960).

Pettengill, G. H., Results as reported to J. V. Evans, Lincoln Lab. Rep. 3G-0004 (1961).

Senior, T. B. A., and K. M. Siegel, Radar reflection characteristics of the moon, Paris Symp. on Radio Astron. (ed. R. N. Bracewell) (Stanford Univ. Press, 1959).

Senior, T. B. A., and K. M. Siegel, A theory of radar scattering by the moon, J. Research NBS 64D, 217 (1960).

Trexler, J. I., Lunar radio echoes, Proc. IRE 46, 286 (1958).

Winter, D. F., Thesis, Harvard University (1961).

Yaplee, B. S., R. H. Bruton, K. J. Craig, and N. G. Roman, Radar echoes from the moon at a wavelength of $10 \mathrm{~cm}$, Proc. IRE 46, 293 (1958). 